\title{
Three Modes of Metal-Enriched Star Formation at High Redshift
}

\author{
Britton D. Smith ${ }^{1}$, Matthew J. Turk ${ }^{2}$, Steinn Sigurdsson ${ }^{3}$, \\ Brian W. O'Shea ${ }^{4}$, and Michael L. Norman ${ }^{5}$ \\ ${ }^{1}$ Center for Astrophysics \& Space Astronomy, Department of Astrophysical \& Planetary \\ Sciences, University of Colorado, Boulder, CO, 80309 \\ email: brittons@origins. colorado.edu \\ ${ }^{2}$ Kavli Institute for Particle Astrophysics and Cosmology, 2575 Sand Hill Rd., Mail Stop 29, \\ Menlo Park, CA 94025 \\ email: mturk@slac.stanford.edu, \\ ${ }^{3}$ Department of Astronomy \& Astrophysics, 525 Davey Laboratory, The Pennsylvania State \\ University, University Park, PA 16802 \\ email: steinn@astro.psu.edu, \\ ${ }^{4}$ Department of Physics \& Astronomy, Michigan State University, East Lansing, MI 48824 \\ email: bwoshea@lanl.gov \\ ${ }^{5}$ Center for Astrophysics and Space Sciences, University of California at San Diego, \\ La Jolla, CA 92093 \\ email: mlnorman@ucsd.edu
}

\begin{abstract}
It is generally accepted that the very first stars in the universe were significantly more massive and formed much more in isolation than stars observed today. This suggests that there was a transition in star formation modes that was most likely related to the metallicity of the star-forming environment. We study how the addition of heavy elements alters the dynamics of collapsing gas by performing a series of numerical simulations of primordial star formation with various levels of pre-enrichment, using the adaptive mesh refinement, hydrodynamic $+\mathrm{N}$-body code, Enzo. At high redshifts, the process of star formation is heavily influenced by the cosmic microwave background (CMB), which creates a temperature floor for the gas. Our results show that cloud-collapse can follow three distinct paths, depending on the metallicity. For very low metallicities $\left(\log _{10}\left(Z / Z_{\odot}\right)<-3.5\right)$, star formation proceeds in the primordial mode, producing only massive, singular objects. For high metallicities $\left(\log _{10}\left(Z / Z_{\odot}\right)>-3\right)$, efficient cooling from the metals cools the gas to the CMB temperature when the core density is still very low. When the gas temperature reaches the CMB temperature, the core becomes very thermally stable, and further fragmentation is heavily suppressed. In our simulations with $\log _{10}\left(Z / Z_{\odot}\right)>-3$, only a single object forms with a mass-scale of a few hundred $M_{\odot}$. We refer to this as the CMBregulated star formation mode. For metallicities between these two limits $\left(-3.5<\log _{10}\left(Z / Z_{\odot}\right)\right.$ $<-3)$, the gas cools efficiently, but never reaches the CMB temperature. In this mode, termed the metallicity-regulated star formation mode, the minimum gas temperature is reached at much higher densities, allowing the core to fragment and form multiple objects with mass-scales of only a few $M_{\odot}$. Our results imply that the stellar initial mass function was top-heavy at very high redshift due to stars forming in the CMB-regulated mode. As the CMB temperature lowers with time, the metallicity-regulated star formation mode (producing multiple low-mass stars) operates at higher metallicities and eventually becomes the sole mode of star formation.
\end{abstract}

Keywords. stars: formation, (cosmology:) early universe

\section{Simulations}

We perform a series of 24 primordial star formation simulations using the Eulerian adaptive mesh refinement hydrodynamics + N-body code, Enzo (Bryan \& Norman 1997, 
Table 1. Summary of simulations performed. All simulations in each set have identical initial conditions. $z_{c o l}$ is the redshift of collapse for the metal-free run in that set. The final column lists the metallicities of all runs performed in that set. In this work, each simulation is referred to by the letter ' $\mathrm{r}$ ', then the number of the initial conditions, followed by the letter ' $\mathrm{Z}$ ' and the $\log$ of the metallicity. The metal-free runs are referred to with the letters ' $m f$ '. For example, run r2_Z-3.5 refers to the simulation with $Z=10^{-3.5} Z_{\odot}$ and using the second set of initial conditions.

\begin{tabular}{ccl}
\hline Set & $z_{c o l}$ & $\log _{10}\left(Z / Z_{\odot}\right)$ \\
\hline 1 & 14.8 & Metal-free, $-6,-5,-4.25,-4,-3.75,-3.5,-3.25,-3,-2.5,-2$ \\
\hline 2 & 17.4 & Metal-free, $-4,-3.5,-3,-2.5,-2$ \\
\hline 3 & 23.9 & Metal-free, $-4,-3.5,-3,-2.5,-2,-2^{*}$ \\
\hline
\end{tabular}

Notes:

${ }^{*}$ This run was performed with $Z=10^{-2} Z \odot$, but without the CMB.

O'Shea et al. 2004). Excluding our three metal-free control runs, the gas in each simulation is homogeneously pre-enriched to some non-zero metallicity. The nature of the initial conditions for our simulations are identical to those used in Smith \& Sigurdsson (2007). All of the simulations have the following parameters: box size of $300 h^{-1} \mathrm{kpc}$, initial redshift, $z_{i}=99, \Omega_{M}=0.3, \Omega_{\Lambda}=0.7, \Omega_{B}=0.04$, Hubble constant, $h=0.7$, in units of $100 \mathrm{~km} \mathrm{~s}^{-1} \mathrm{Mpc}^{-1}$, and $\sigma_{8}=0.9$. We use three different sets of initial conditions, each with the parameters described above, but created with unique random seeds. The first set is the one used by Smith \& Sigurdsson (2007). The second and third sets were initially used by O'Shea \& Norman (2007) and correspond to the runs named L0_30A and L0_30D in that work. A short list of the simulations performed is given in Table 1 .

We use the second implementation of the optically-thin metal cooling method of Smith et al. (2008). This method uses tabulated cooling functions created with the photoionization software, Cloudy (Ferland et al. 1998), for all elements heavier than He, up to atomic number $30(\mathrm{Zn})$. A solar abundance pattern is used for the metals in all of the simulations.

\section{Results}

The gas phase critical metallicity, $Z_{c r}$, has been estimated analytically by calculating the chemical abundance required for the cooling time to equal the dynamical time at the minimum temperature reached in the collapse of metal-free gas, $n \sim 10^{3-4} \mathrm{~cm}^{-3}$ and $T$ $\sim 200 \mathrm{~K}$ (Bromm \& Loeb 2003, Santoro \& Shull 2006). Since this temperature minimum occurs at slightly different temperatures and densities for each of the three metal-free runs (see the solid, black curves in Figure 3), we calculate individual values of $Z_{c r}$ for each run. The values of $Z_{c r}$ for each run are: Set $1,10^{-4.08} Z_{\odot}$; Set $2,10^{-3.90} Z_{\odot}$; and Set $3,10^{-3.85} Z_{\odot}$.

In Figure 1, we show projections of baryon density of the central 0.5 pc surrounding the point of maximum baryon density for all runs in Set 1. For the runs with metallicities near or below $Z_{c r}\left(10^{-4.08} Z_{\odot}\right.$ for this set of simulations $)$, the central cores appear quite round and show no clear signs of forming more than one object. In the metallicity range from $10^{-3.75} Z_{\odot}$ to $10^{-3.25} Z_{\odot}$, the cores appear increasing asymmetric, with at least one additional density maximum present. However, at metallicities at or above $10^{-3} Z_{\odot}$, the cores return to a more spherical shape.

In Figure 2, we plot a histogram of all the number of clumps found in each run, as a function of the metallicity of the run. Figure 2 confirms what is seen in Figure 1. In all 


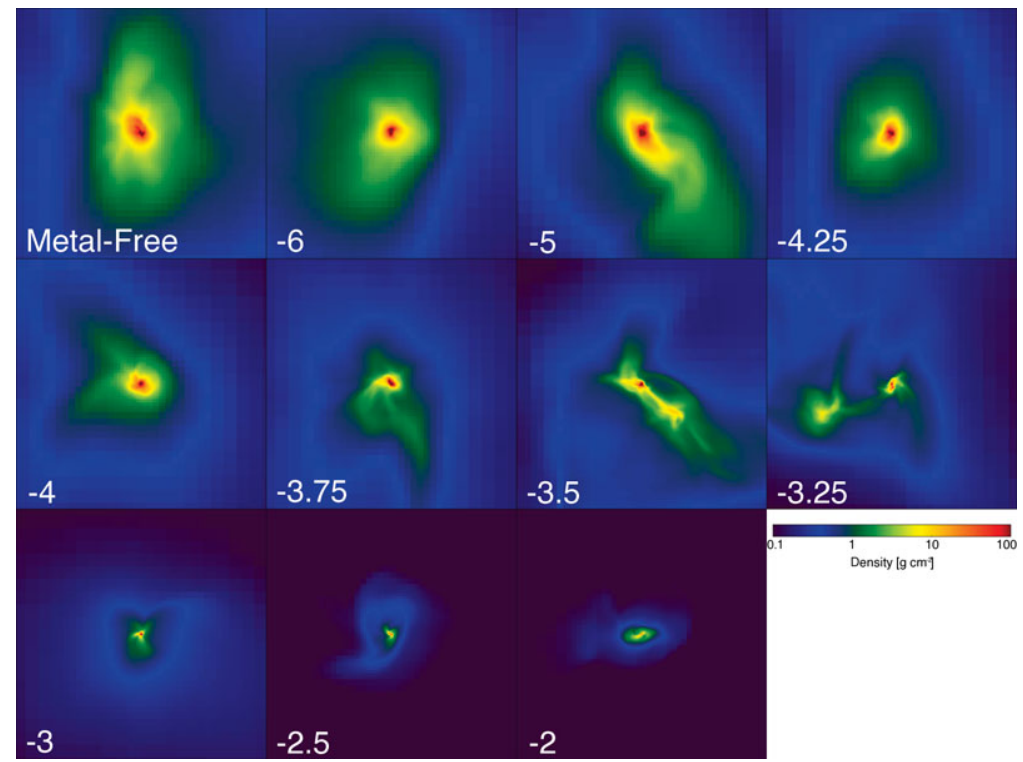

Figure 1. Density projections for the final output of all runs in Set 1. Each projection is centered on the location of maximum density in the simulation box and has a width of $0.5 \mathrm{pc}$ proper. The labels in each panel indicate the log of the metallicity with respect to solar for that run.

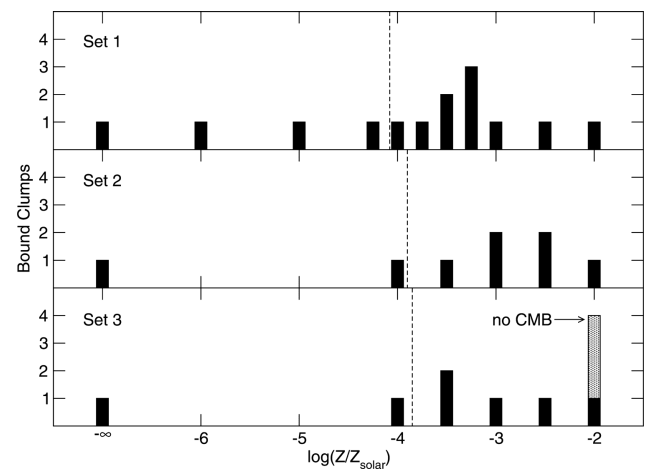

Figure 2. The number of bound clumps found within a sphere of radius $1 \mathrm{pc}$, centered on the point of maximum density, as a function of metallicity for all of the simulations performed. The grey bar in the bottom panel denotes the run where the temperature floor created by the CMB was not included. In each panel, the vertical dashed line represents the estimated value of $Z_{c r}$ for each set.

runs with metallicities below $Z_{c r}$, only a single bound clump is found. As the metallicity increases, the number of clumps increases, then decreases back to only a single clump for the highest metallicities. The range of metallicities where fragmentation occurs is consistent between Sets 1 and 3, but offset by 0.5 dex toward higher metallicities for Set 2. It is not clear what causes this offset, but the qualitative trend of increasing and then decreasing number of clumps exists in all 3 sets. It is also worthwhile to note that runs r1_Z-4, r1_Z-3.75, and r2_Z-3.5, while slightly above $Z_{c r}$, do not show fragmentation.

In Figure 3, we plot the number density vs. gas temperature for the final output of each simulation in Sets 1 and 3. In all the runs with $Z<Z_{c r}$, the cooling is too low to prevent the temperature from rising at the $\mathrm{H}_{2}$ thermalization density, $n \sim 10^{4} \mathrm{~cm}^{-3}$. Therefore, the minimum fragmentation mass for these runs, set by the Jeans mass as the temperature 

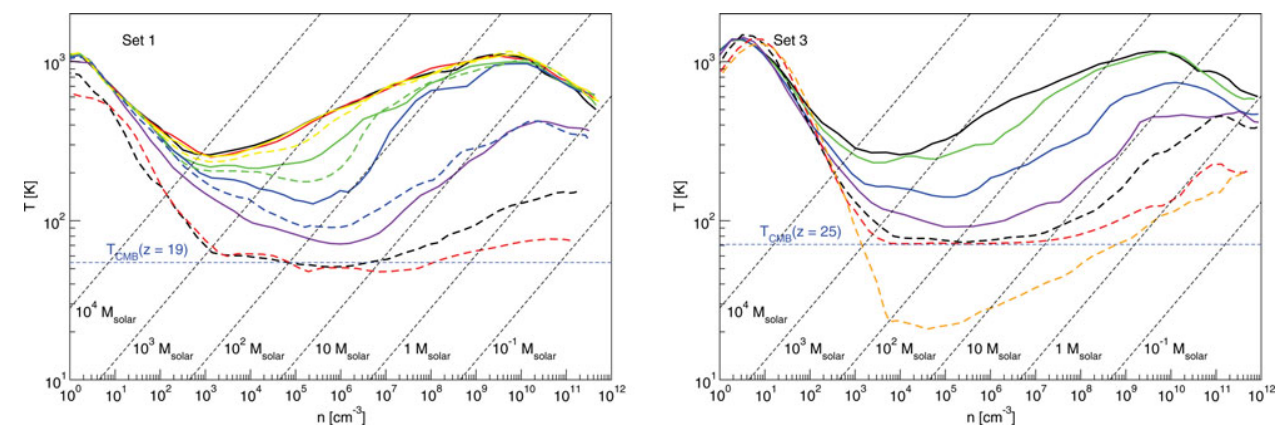

Figure 3. Mass-weighted, average gas temperature as a function of number density for all runs in Set 1 (left-panel) and all runs in Set 3 (right-panel). The metallicities are $Z=$ 0 (solid-black), $10^{-6} Z_{\odot}$ (solid-red), $10^{-5} Z_{\odot}$ (yellow), $10^{-4.25} Z_{\odot}$ (dashed-yellow), $10^{-4} Z_{\odot}$ (green), $10^{-3.75} Z_{\odot}$ (dashed-green), $10^{-3.5} Z_{\odot}$ (blue), $10^{-3.25} Z_{\odot}$ (dashed-blue), $10^{-3} Z_{\odot}$ (purple), $10^{-2.5} Z_{\odot}$ (dashed-black), $10^{-2} Z_{\odot}$ (dashed-red), and $10^{-2} Z \odot$ with the $\mathrm{CMB}$ removed (orange-dashed). The thin, black, dashed lines indicate lines of constant Jeans mass. The horizontal, blue, dashed lines denote the CMB temperature at the approximate redshifts of collapse.

minimum, is well over $1,000 M_{\odot}$, which is nearly equivalent to the total enclosed mass. Even though runs r1_Z-4 and r1_Z-3.75 are above $Z_{c r}$, the additional cooling provided by the metals is not sufficient to significantly lower the minimum fragmentation mass. For runs r1_Z-3.5 and r1_-3.25, the more efficient cooling lowers the minimum fragmentation mass to just over $100 M_{\odot}$, which is approximately a factor of 10 lower than the total mass within 1 pc.

For the runs with the highest metallicities, as in runs r1_Z-2.5 and r1_Z-2, the gas cools all the way to the temperature of the CMB. The cooling proceeds so efficiently that the gas has not had sufficient time to reach high densities before hitting the temperature floor of the CMB. Fragmentation can only continue as long as the temperature decreases with increasing density (Larson 1985, Larson 2005). Although the temperature decreases slightly in runs r1_Z-2.5 and r1_Z-2 for densities greater than $10^{3} \mathrm{~cm}^{-3}$, the temperature minimum is effectively at $n=10^{3} \mathrm{~cm}^{-3}$, where the gas reaches the CMB temperature. Near the CMB temperature, the value of the cooling rate, $\Lambda$, effectively becomes $\left(\Lambda(T)-\Lambda\left(T_{C M B}\right)\right)$. Therefore, when the gas reaches the CMB temperature, the cooling rate drops to zero, and the cooling time becomes infinite. The gas cloud becomes extremely thermally stable, preventing further fragmentation. To verify that the CMB is indeed suppressing fragmentation, we run one simulation, r3_Z-2_noCMB, with the CMB temperature floor removed. With the CMB temperature floor in place, r3_Z-2 formed a single bound clump. When the CMB was removed, the gas was able to cool to roughly 20 $\mathrm{K}$ (dashed, orange curve in right panel of Figure 3) and form 4 bound clumps (Figure 2).

\section{Discussion}

The mass scale of collapsing clumps can be estimated from the Jeans mass at the end of the cooling phase. This implies the existence of three distinct metallicity regimes for star formation. In the first regime, $Z<Z_{c r}$, which we refer to as the 'primordial' mode, metals do not provide enough additional cooling to allow the gas temperature to continue to decrease monotonically with increasing density when the core reaches the $\mathrm{H}_{2}$ thermalization density. In this case, the collapse proceeds in a similar way to the metalfree scenario, resulting in the formation of a single, massive object. At the other extreme, we define $Z_{C M B}$ as the metallicity at which the gas can cool to the CMB temperature. 
When $Z \gg Z_{C M B}$, the cloud-core will efficiently cool to the temperature of the CMB when the central density is still relatively low. In this scenario, fragmentation is limited by cooling rapidly to the CMB temperature, as the mass scale is determined by the Jeans mass at the density when the core first reaches the CMB temperature. We refer to this as the CMB-regulated star formation mode, similar to Tumlinson (2007). As fragmentation is severely limited in this mode, these stars will most likely be more massive on average than the characteristic mass of stars forming today. Finally, our simulations have shown that there exists a special range in metallicity, $Z_{c r} \leqslant Z<Z_{C M B}$, where the core does not reheat at the metal-free stalling point, but also cannot cool all the way to the CMB temperature. The minimum temperature is set only by the balance of radiative cooling and adiabatic heating. The mass scale is not regulated externally by the CMB, but rather internally by the metallicity-dependent gas-cooling. Hence, we term this the metallicityregulated star formation mode. This mode produces the lowest mass stars of the three modes mentioned.

The CMB-regulated star formation mode creates a means by which a higher number of massive stars are formed in the very early universe, when the CMB temperature was much higher. As the universe evolves, the CMB temperature will slowly decrease, which will increase the metallicity required to reach the CMB temperature, referred to here as $Z_{C M B}$. The decrease in the CMB temperature also means that the fragmentation mass scale will be lower at the point where the gas reaches the cooling floor. Thus, the characteristic mass of stars produced by the CMB-regulated mode will slowly decrease with time. Such a phenomenon was predicted by Larson(1998). As the metallicity threshhold for the CMB-regulated mode advances to higher metallicity, the range of operation of the metallicity-regulated mode will extend to take its place. Observations of nearby starforming clouds show that the minimum achievable temperature in the local universe is roughly $10 \mathrm{~K}$ (e.g., Evans(1999)). This implied that the CMB-regulated star formation mode is in operation up to $z \sim 2.7$, at the absolute latest.

\section{References}

Bromm, V. \& Loeb, A. 2003, Nature, 425, 812

Bryan, G. \& Norman, M. L. 1997, in Workshop on Structured Adaptive Mech Refinement Grid Methods, ed. N. Chrisochoides, IMA Volumes in Mathematics No. 117 (Springer-Verlag)

Evans, II, N. J. 1999, ARAA, 37, 311

Ferland, G. J., Korista, K. T., Verner, D. A., Ferguson, J. W., Kingdon, J. B., \& Verner, E. M. 1998, PASP, 110, 761

Larson, R. B. 1985, MNRAS, 214, 379

-. 1998, MNRAS, 301, 569

—. 2005, MNRAS, 359, 211

O'Shea, B. W., G., B., Bordner, J., Norman, M. L., Abel, T., Harknes, R., \& Kritsuk, A. 2004, in Lecture Notes in Computational Science and Engineering, Vol. 41, Adaptive Mesh Refinement - Theory and Applications, ed. T. Plewa, T. Linde, \& V. G. Weirs

O'Shea, B. W. \& Norman, M. L. 2007, ApJ, 654, 66

Santoro, F. \& Shull, J. M. 2006, ApJ, 643, 26

Smith, B., Sigurdsson, S., \& Abel, T. 2008, MNRAS, 385, 1443

Smith, B. D. \& Sigurdsson, S. 2007, ApJ1, 661, L5

Tumlinson, J. 2007, ApJl, 664, L63 